\title{
3D Antenna Array for SWIPT Sensing with WPT Capabilities
}

\author{
Diogo Pires ${ }^{1}$, Daniel Belo ${ }^{1}$, Marina Jordão ${ }^{1}$, Pedro Pinho ${ }^{2}$, Nuno Borges de Carvalho ${ }^{1}$, \\ ${ }^{1}$ Instituto de Telecomunicações, Dep. Electrónica, Telecomunicações e Informática, \\ Universidade de Aveiro, Aveiro, Portugal, drppires@ua.pt \\ ${ }^{2}$ Instituto Superior de Engenharia de Lisboa, Lisboa, Portugal
}

\begin{abstract}
In this work, the design and development of an alternative three-dimensional array is presented. This arrangement aims to improve Simultaneously Wireless Information and Power Transfer (SWIPT) systems and to provide advantages when integrated into a Wireless Sensor Network (WSN) architecture. The conceived 3D antenna array consists of eight antenna elements operating at $5.65 \mathrm{GHz}$ that are attached in a 3D printed heptagonal prism. With this structure, it is intended to achieve as close as possible to an omnidirectional radiation pattern with considerable gain, avoiding power losses. The experimental measurements carried out are in line with the performed electromagnetic simulations and validate the array operation. A full azimuth coverage was ensured with an average realized gain of $6.7 \mathrm{dBi}$. For some azimuth directions, this gain can reach approximately $8.35 \mathrm{dBi}$. This array proves to be a reliable solution to fed multiple low-power sensors that are placed over the 360 azimuth angles.
\end{abstract}

Index Terms - 3D Antenna Arrays, Characterization, Azimuth Coverage, Wireless Power Transmission, Wireless Sensor Network.

\section{INTRODUCTION}

The emerging concept of the Internet of Things (IoT) networks will boost the smart cities concept to a real panorama, which will involve extensive use of sensors. The massive deployment of the sensors will lead to a considerable amount of wasted batteries which is a serious threat to the public health and to the environment. The development of passive or low-power sensors can be seen as a solution to this problem and as a small step to a sustainable future. However, it is necessary to find an efficient system to feed all these sensors. In order to solve this problem, the optimization of Wireless Power Transmission (WPT) systems and energy harvesting technologies have been areas of high research, since it is expected that it will become a valid alternative to feed those sensors, improving these systems architectures and minimizing the related environmental issues [1]. Moreover, the implementation of these techniques can solve adversities of such applications where the batteries removal would be an advantage, such as space exploration, where the batteries replacement can represent a hard task.

Three-dimensional (3D) antennas arrays that rest in quasispherical structures, can be explored in order to verify if they can present a valid alternative to accomplish these demands since they can radiate to several places. In this way, they have the capability to supply several sensors scattered over a wide area without a mechanical moving and are easy to attach in every place. However, unfortunately, with these antennas, there are some emerging problems due to the high complexity in terms of design and implementation. Furthermore, since the array factor is not linear in these structures, some adversities may occur when it is intended to achieve steering capabilities.

The implementation of 3D antenna arrays in different scenarios have been studied in the past years. In [2] and [3] 3D antenna arrays were proposed by the same authors, for maritime and satellite communications, respectively. In [2] due to the near-spherical shape, the antenna gain is constant while the beam is moving over the hemisphere, even in low elevation angles. In [3], the proposed arrangement presents the particularity of ensuring the hemispherical coverage by combining the patterns produced for the single elements individually with the ones obtained by the combination of two active elements. Localization systems are another application that would benefit from these 3D arrays coverage capabilities, since they can be easily placed on top of any indoor space. In [4], an antenna array attached on a 3D shape was described for indoor position estimation systems.

The main goal of this work is to project a 3D array capable of performing a robust coverage over all the azimuth angles, minimizing the gain variation. In this way, the energy can be oriented to several target locations with stable Effective Radiated Power.

This paper is organized as follows. In Section II, the designed 3D antenna array is described in detail. In order to validate the antenna array frequency operation and its radiation pattern simulations were performed and presented in Section III. In Section IV, it will be described several measurements that were carried out in order to validate both the antenna elements and the 3D array operation. Finally, the obtained results will be analyzed and the conclusions will be drawn in Section V.

\section{3D Antenna ARray: DESIGN}

The main motivation is that these structures, besides being easy to install in almost any place, are capable of receive and transmit signals/power in all azimuth directions, which makes them capable of providing multiple targets, such as sensors or communication devices. In this case, it is intended that the designed array can ensure a robust coverage over the $360^{\circ}$ 

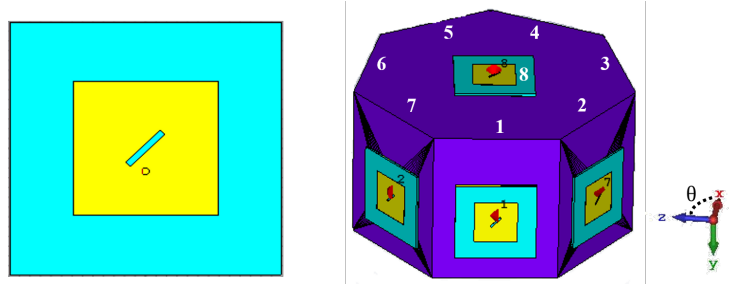

Fig. 1. Single element and 3D antenna array.

azimuth plane and also, through WPT, supply several targets scattered around itself.

Having this coverage characteristics as main requirement, the supporting structure must allow the attachment of enough antenna elements for the purpose. So, the chosen geometry for the structure was a heptagonal prism since allows the employment of seven elements in the side faces (enough to radiate over all the hemisphere) not too separated from each other. Due to this proximity, it is possible to originate beams produced from the combination of two consecutive elements. In its turn, the top element has the objective to cover the plane above it, while the remaining seven elements (separated by $0.69 \lambda_{0}$ from each other) are intended to cover the azimuth plane. In Fig. 1 is depicted both the designed antenna element and the 3D antenna array.

The arrangement was designed using the SolidWorks software and printed in poly-lactic acid (PLA) from the Ultimaker supplier (dielectric constant $\epsilon_{r}=2.7$ and dissipation factor, $\operatorname{tg}(\delta)=0.008 @ 1 \mathrm{MHz}$ ). The antenna array elements (microstrip patch antennas), were designed on Isola IS680 substrate $\left(\epsilon_{r}=3.45, \mathrm{~h}=0.762 \mathrm{~mm}\right)$. In the future it is intended to test this array operation not only in WPT applications but also in wireless communication systems. For this reason, the antennas were circularly polarized, aiming to avoid power losses that can occur from a polarization misalignment between the transmitting and receiving antennas. Circular polarization can be obtained by changing either the antenna's physical structure or their feeding form in order to excite two orthogonal modes with a $90^{\circ}$ time-phase difference between them. In this case, a Right Hand Circular Polarization (RHCP) was achieved by cutting a slot in the square patch center [5]. Simulations were performed using Computer Science Technology (CST) software in order to validate the antenna's operating frequency. In Fig. 2 is presented the antenna's reflection coefficient. It can be concluded that the antenna presents an good match for the desired frequency since their $S_{11}$ parameter has -28.18 $\mathrm{dB}$ of magnitude at $5.65 \mathrm{GHz}$. To evaluate if the antenna is operating with circular polarization, the antenna's radiation pattern was also analyzed. The main features (realized gain and axial ratio) were simulated. In Fig. 3 are depicted the obtained results. It can be concluded that the circular polarization was achieved, since its axial ratio is lower than $3 \mathrm{~dB}$ for the angles of maximum radiation intensity.

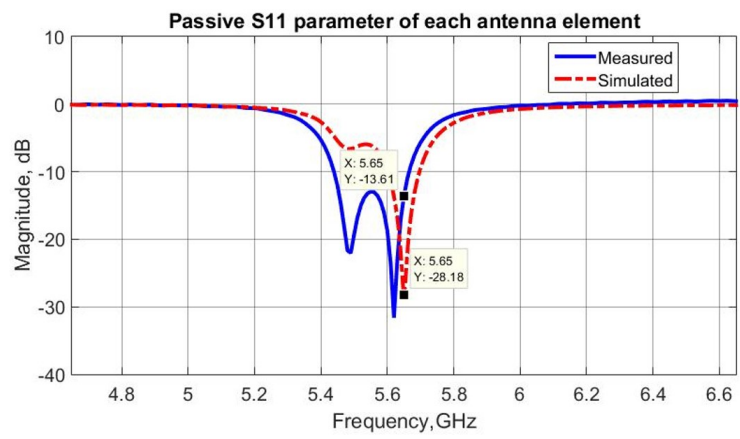

Fig. 2. Antenna element: simulated and measured $S_{11}$ parameter.

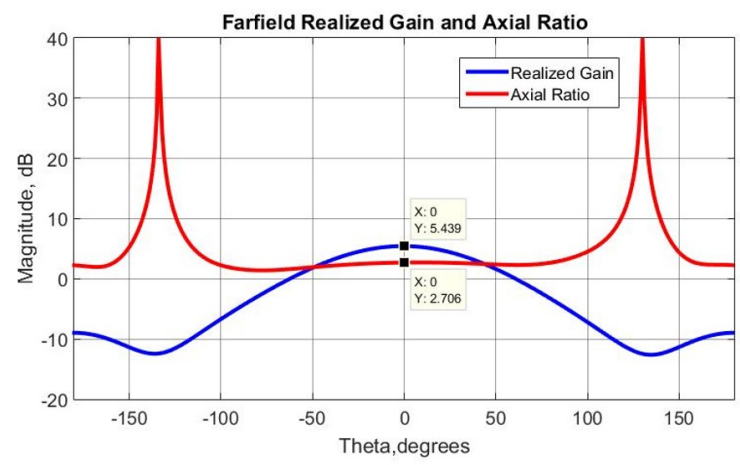

Fig. 3. Antenna element realized gain and axial ratio.

\section{3D ANTENNA ARRAY: PERformed Simulations}

As previously mentioned, this array design comes with the primary goal of being able to establish radio links over the $360^{\circ}$ azimuth angles without moving, and so, supply several passive/low-power sensors, which will be scattered around it. A beam-switch approach is intended to be followed, which means that the array will never operate with all elements active but only with the necessary ones to transmit a signal to a specific direction. It is expected that by switching between the array elements that are active, a full azimuth coverage can be achieved. Simulations were performed in order to evaluate the overall radiation capabilities. Two types of radiation pattern were considered: the ones produced by each individual element and those that result from the combination of two active sequential elements. The results are depicted in Fig. 4. It can be concluded that, by switching between the different radiation patterns, it is possible to keep a similar signal strength over the $360^{\circ}$, ensuring full azimuth coverage. However, when two elements are active simultaneously, their electromagnetic fields constructively interfere enhancing the power radiated to the desired directions. This particularity produces a narrower beam that confers a higher gain than when only one element is active.

There are some positions where the signal quality in the receiver presents some gain degradation. An example of those positions is when the target is placed at a far-field position in the angles that fall between the antenna elements. When 


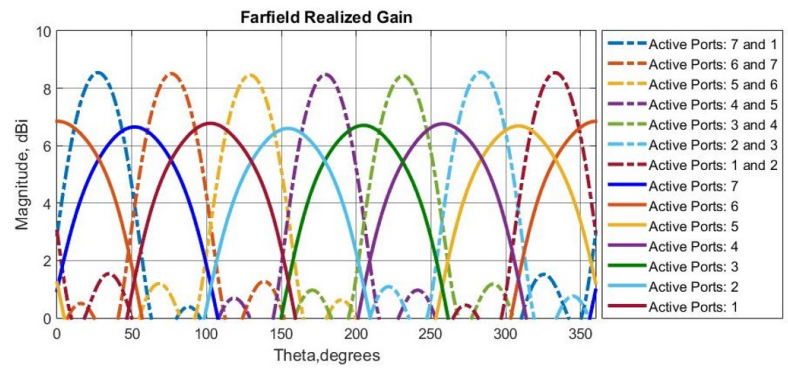

Fig. 4. Simulated radiation patterns

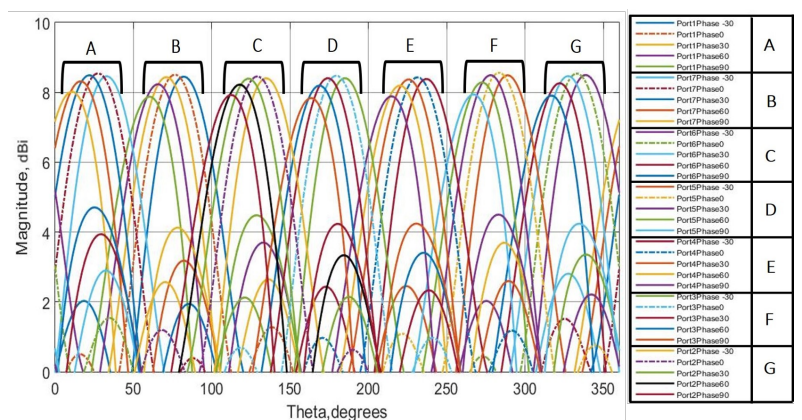

Fig. 5. Phase sweep impact on the radiation pattern produced by two consecutive elements when active simultaneously.

this happens, the receiver is not aligned with the direction of maximum radiation intensity. A solution to establish a link is through a beam produced by the combination of two sequential active elements simultaneously. For this reason, it was considered essential to understand how this beam can be controlled in order to improve the signal quality when the receiver is in these positions. This analysis also provides essential insights if in the future, beam-steering capabilities are intended to be introduced in this system. A phase sweep analysis was then performed when two ports are simultaneously active. One port phase shift was fixed at $0^{\circ}$ while the other one was swept from $0^{\circ}$ to $90^{\circ}$ with a $30^{\circ}$ step. The obtained results are depicted in Fig. 5.

Even with the phase shifts introduced, the signal gain remains stable, and the phase variations introduced cause, as expected, a beam shift. This analysis makes it possible to estimate which phase shifts should be introduced in order to ensure the strengthened signal to a receiver position.

\section{Performed Measurements: Results And DISCUSSION}

After having the antenna's prototype it is necessary to evaluate their reflection coefficient for the operating frequency. Using a Vector Network Analyzer (VNA), the antenna's $S_{11}$ parameter was measured. The results are presented in Figure 2. Despite the differences between the simulated and measured results, the antenna operation was validated for the desired frequency.

In order to evaluate the real coverage that the $3 \mathrm{D}$ antenna array can achieve, measurements in the anechoic chamber

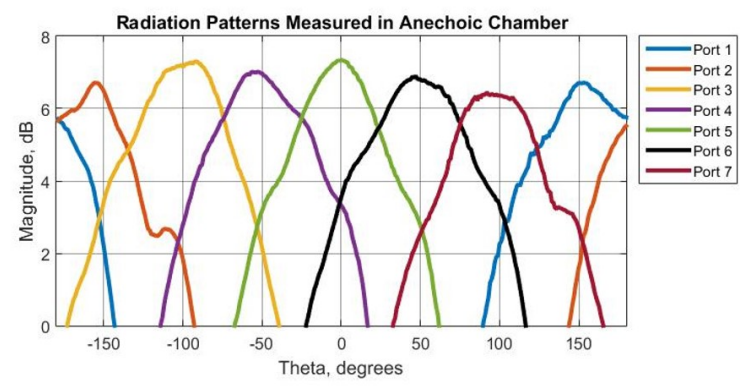

Fig. 6. Radiation patterns measured in anechoic chamber.

were performed. The radiation pattern of each of the seven elements was measured individually and are presented in Fig. 6. It is possible to conclude that although the jitter that should occur due to the antenna rotation motion inside the chamber, a small signal's gain variations occur between different the main lobes. This means that by switching between the active array elements, a full azimuth coverage is ensured, and the antenna gain is approximately constant.

\section{CONCLUSION}

In this work, the design and characterization of a 3D antenna array was presented. This alternative arrangement, which can easily be printed in a 3D printer (low-cost), aims to improve WPT systems as well as WSNs. The main goal was achieved since the array, that followed a beam-switch approach, proved to be capable of a full azimuth plane coverage, being able to transmit a signal with considerable gain to any far-field position around itself. Thus, the developed 3D array is capable of transmitting energy to a sensor, regardless of its location. This arrangement also allows the radio link adjustment without mechanical movement, which has advantages in applications where the receiver has a fixed position.

\section{ACKNOWLEDGMENT}

The work of Diogo Pires was supported by the European Regional Development Fund (FEDER), through the Competitiveness and Internationalization Operational Programme (COMPETE 2020) of the Portugal 2020 framework [Project TexBoost with Nr. 024523 (POCI-01-0247- FEDER-024523)]. The work of Daniel Belo was supported by the Fundação para a Ciência e Tecnologia (F.C.T.) under Ph.D. Grant SFRH/BD/142403/2018. The work of Marina Jordão was supported by the Fundação para a Ciência e Tecnologia (F.C.T.) under Ph.D. Grant SFRH/BD/143204/2019.

\section{REFERENCES}

[1] N. Borges Carvalho et al., "Wireless Power Transmission: R\&D Activities Within Europe," in IEEE Transactions on Microwave Theory and Techniques, vol. 62, no. 4, pp. 1031-1045, April 2014.

[2] M. Geissler, M. Bottcher, R. Gieron, M. Eube and P. Siatchoua, "A Low-Cost Phased Array for Mobile Satellite Communications," 2007 2nd International ITG Conference on Antennas, Munich, 2007, pp. 148152. 
[3] M. Geissler et al., "L-band phased array for maritime satcom," 2010 IEEE International Symposium on Phased Array Systems and Technology, Waltham, MA, 2010, pp. 518-523.

[4] A. Cidronali, S. Maddio, G. Giorgetti and G. Manes, "Analysis and Performance of a Smart Antenna for 2.45-GHz Single-Anchor Indoor Positioning," in IEEE Transactions on Microwave Theory and Techniques, vol. 58, no. 1, pp. 21-31, Jan. 2010.

[5] C.A. Balanis. Antenna Theory: Analysis and Design. Wiley, 2016. 